ermöglichen will, und da alle wirklichen Sterpe bei der Messung abzuschwächen sind, so ist klar, dass bei jedem Fernrohr eine gewisse untere Helligkeitsgrenze nicht liberschritten werden kann. Bei dem hiesigen Grubb'schen Refractor von $209 \mathrm{~mm}$ Objectivöffnung darf man mit dem neuen Photometer etwa bis zu Sternen 8. Grösse gehen.

Zur Bestimmung der Keilconstanten, von deren Zuverlässigkeit in erster Linie die Messungsresultate abhängen, kann man mit Vortheil von der Combination der beiden Messungsprincipe Gebrauch machen. Man stellt zu diesem Zweck den Intensitätskreis zunächst auf eine bestimmte $\mathrm{Ab}$ lesung, etwa $10^{\circ}$, ein, wählt dann irgend einen wirklichen Stern und bringt ihn mit Hülfe des Keils auf die gleiche Helligkeit mit dem künstlichen; dann stellt man den Intensitätskreis auf eine andere Ablesung, etwa $50^{\circ}$, ein und macht den ausgewählten wirklichen Stern durch Verschiebung des Keils abermals gleich dem kitnstlichen. Die Differenz der entsprechenden Keilablesungen giebt in Verbindung mit der am Intensitätskreis hervorgebrachten Helligkeitsänderung einen Węrth für die Keilconstante. Man sieht ohne weiteres, dass es durch entsprechende Wahl der Einstellungen am Intensitätskreis, sowie durch Benutzung verschieden heller und verschieden gefärbter wirklicher Sterne leicht ist, den Keil an jeder beliebigen Stelle und für jede Sternfarbe, so genau wie man will, zu untersuchen.

Es verdient noch hervorgehoben zu werden, dass das Instrument jeder Zeit, nach Herausschrauben des Keils, als gewöhnliches Zöllner'sches Photometer benutzt werden kann, und dass es andererseits auch, sobald man die Lampe fur die künstlịchen Sţerne ausser Thätigkeit lässt, als blosses Keilphotometer nach dem Auslöschungsprincip dienen kann. Die neue Form ist also gewissermaassen ein Universal. photometer, welches nicht nur die getrennte Anwendung zweier Messungsprincipe gestattet, sondern durch die Com. bination auch die Möglichkeit gewährt, dieselben unmittelbar mit einander zu vergleichen; und ausserdem durch Einführung einer meuen Messungsmethode gewisse Auffassungsfehler zu vermeiden.

Potsdam, Astrophysikalisches Observatorium, I900 Dec. 19.

G. Miiller.

\title{
Ueber die Abhängigkeit der Grössenschätzungen auf photographischen Aufnahmen vom Abstand von der Plattenmitte.
}

\author{
Von $\mathcal{F}$. Scheiner.
}

In der Einleitung zu dem soeben erschienenen II. Bande der Photogr. Himmelskarte sind Untersuchungen enthalten über die Beeinflussung der Grössenschätzungen durch die normale Distorsion der Objective. Obgleich diese Beeinflussung für verschiedene Objective und auch für verschiedene Beobachter durchaus nicht die gleiche ist, dürfte eine Untersuchung derselben in einem speciellen Falle doch allgemeineres Interesse beanspruchen, und ich möchte dieselbe daher auch an dieser Stelle mittheilen.

In welcher Weise die Grössenbestimmungen bei photographischen Aufnahmen durch Messung von dem Abstande von der Plattenmitte abhängen, habe ich schon früher gezeigt (Photographie der Gestirne, S. 226). Für den hiesigen Photographischen Refractor ist bis zu einem Abstande von $30^{\circ}$ von der Mitte von einer Aenderung der Durchmesser der Sternscheibchen nichts zu erkennen; von da ab aber wird dieselbe merklich und bewirkt, dass schliesslich bei $60^{\circ}$ Abstand die Sterne um 0.25 Grössenclassen zu hell gemessen werden. Bei schwächeren Sternen, bei denen die Mitte des Scheibchens nicht ausexponirt ist, tritt mit grösserem Abstande von der Mitte umgekehrt die Tendenz ein, die Helligkeit zu gering zu schätzen, und die schwächsten Sterne, die in der Mitte der Platte als I I.0 taxirt werden, sind am Rande der Platte überhaupt nicht mebr zu sehen. Auf diesen Umstand ist beim Einuiben der Beobachter im Grössenschätzen besondere Aufmerksamkeit verwendet worden, indem die Randsterne von den übrigen getrennt mit den Grössen der BD. verglichen wurden. Es sollte dadurch eine gleichmässige Scala uber die ganze Platte hinuber erreicht werden.
Um das bis jetzt vorliegende Material der Grössenschätzungen in dieser Beziehung zu prüfen, habe ich folgendes Verfahren eingeschlagen. Nach den internationalen Abmachungen decken sich benachbarte Platten aus den verschiedenen Declinationsgraden immer in der Weise, dass die Mitte der einen Platte auf eine der Ecken der anderen zu liegen kommt. Bildet man nun auf den gemeinschaftlichen Plattenvierteln die Differenzen der Grössenschätzungen der identischen Sterne, so setzen sich diese Differenzen zusammen aus einer constanten Abweichung zwischen den beiden Platten, die auf der Verschiedenheit der Luftzustände, der Empfindlichkeit der Platten u.s. w. beruht, und aus einem variablen Betrage, der von dem Abstand von den beiden Mittelpunkten abhängt.

Um die Vergleichung möglichst einfach zu gestalten, wurden die Grössendifferenzen in eine vergrösserte Darstellung des betreffenden Gitterviertels eingetragen, und zwar in die Gitterquadrate, in denen sich die Sterne thatsächlich befinden. Alsdann wurden mit dem Zirkel von den beiden Ecken resp. Plattenmittelpunkten aus concentrische Kreisstuicke über diese graphische Darstellung geschlagen mit Abständen von je ro Bogenminuten. Dadurch konnten die Distanzen eines jeden Sterns von den beiden Mittelpunkten unmittelbar abgelesen und den Differenzen zugeschrieben werden. Diese Vergleichung wurde für die drei Grössenabtheilungen 7.5 bis $8.4,8.5$ bis 9.2 und 9.3 bis 11.0 getrennt durchgefuhrt. Die Trennung der beiden letzten Abtheilungen wurde deshalb zwischen die Grösse 9.2 und 9.3 gelegt, weil im allgemeinen oberhalb der Grösse 9.2 die 
Sternscheibchen nicht mehr völlig geschwärzt sind, so dass hier der oben angedeutete Umkehrpunkt in der Wirkung des Abstandes von der Mitte liegen muss.

Zur Vergleichung konnten ungefähr 7600 Grössenschätzungen benutzt werden.

Die Ermittelung einer Function des Abstandes von der Mitte, welche die so gewonnenen Grössendifferenzen möglichst genau darstellt, schien mir bei dem subjectiven Charakter der letzteren keinen $Z$ weck zu haben, und ich habe mich deshalb damit begnligt, bei dem grossen Umfange der Rechnungen eine möglichst einfache Darstellung zu wăhlen, die wenigstens genugt, ein Bild der Verhältnisse zu geben.

Bezeichnet man die Abstände eines Sterns von den beiden Mittelpunkten mit $r_{1}$ und $r_{2}$, die entsprechende Grössendifferenz mit $A$, so sind als einfachste Beziehungen aufzustellen :

$$
\begin{aligned}
& \text { 1) } \Delta=a+b\left(r_{2}-r_{1}\right)+c\left(r_{2}-r_{1}\right)^{2} \\
& \text { 2) } \Delta=a+b e^{\left(r_{2}-r_{1}\right)} \\
& \text { 3) } \Delta=a+b\left(r_{2}-r_{1}\right)
\end{aligned}
$$

Einige Versuche zeigten, dass die einfachste Darstellung durch eine gerade Linie nicht merklich schlechter ausfiel, als durch die beiden Curven, von denen ja die zweite entschieden der Wahrheit am nächsten kommen wird, und ich habe daher die Constanten $a$ und $b$ für sämmtliche Vergleichungen nach der Methode der kleinsten Quadrate mit Formel (3) berechnet.

Zunächst mögen die Resultate für den Beobachter
Schwassmann folgen, die sich nur auf Band I der Himmelskarte beziehen.

Die Differenzen sind gebildet im Sinne der bei besserer Luft aufgenommenen Platten minus der bei schlechterer erhaltenen, so dass die $a$ im allgemeinen positiv zu erwarten sind. Die mit $n$ überschriebene Columne enthält die Anzahl der benutzten Differenzen; betrug dieselbe weniger als 8 , so

\begin{tabular}{|c|c|c|c|c|c|c|c|c|c|}
\hline & \multicolumn{3}{|c|}{ Grösse I I bis 9.3} & \multicolumn{3}{|c|}{ Grösse 9.2 bis 8.5} & \multicolumn{3}{|c|}{ Grösse 8.4 bis 7.5} \\
\hline Platte Nr. & $a$ & $b$ & $n$ & $a$ & $b$ & $n$ & $a$ & $b$ & $n$ \\
\hline $214-54$ & +0.09 & -0.02 & I 9 & - & - & 5 & - & - & 3 \\
\hline $214-55$ & $+0.6 \mathrm{I}$ & -0.03 & 14 & - & - & 3 & - & 一 & $\circ$ \\
\hline $215-55$ & +0.57 & -0.03 & I 8 & - & - & 5 & - & - & $\mathbf{I}$ \\
\hline $2 \div 5-56$ & +0.98 & -0.03 & 27 & +0.87 & -0.10 & 9 & - & - & 2 \\
\hline $216-56$ & +0.46 & -0.01 & $4 I$ & - & - & 7 & 一 & 一 & I \\
\hline $76-236$ & +0.46 & -0.01 & I I & - & - & 3 & - & - & 1 \\
\hline $197-358$ & +0.09 & +0.02 & 82 & +0.03 & -0.08 & 19 & - & - & 4 \\
\hline $453-613$ & -0.18 & +0.04 & 127 & -0.06 & -0.08 & 24 & -0.36 & -0.09 & 9 \\
\hline $454-6$ I 3 & -0.47 & +0.05 & 90 & -0.43 & -0.05 & 26 & -0.20 & -0.18 & I 3 \\
\hline $5 \times 4-6$ I 4 & $-0.5^{8}$ & +0.04 & 165 & -0.40 & -0.08 & $3^{6}$ & -0.49 & -0.10 & 1 8 \\
\hline $516-677$ & +1.09 & $+0.0 \mathrm{I}$ & 81 & +1.09 & -0.07 & 48 & +0.75 & -0.12 & 9 \\
\hline $83 z-676$ & -0.15 & -0.01 & 405 & +0.10 & -0.03 & 49 & +0.42 & -0.01 & 9 \\
\hline
\end{tabular}
sind die Werthe von $a$ und $b$ als zu unsicher nicht aufgeführt worden.
Die constanten Abweichungen a der Grössenschätzun. gen erreichen ziemlich starke Beträge, im Mittel, ohne Berücksichtigung des Vorzeichens, für die drei Grössenab. theilungen: $0^{\mathrm{m}}{ }_{4} 8, \circ^{\mathrm{m}} 43$ und $\circ^{\mathrm{m}} \cdot 44$, mit einem w. Fehler der einzelnen $a$ von $\pm 00^{\mathrm{m}} \circ 3, \pm 0 \stackrel{\mathrm{m}}{\circ} 06$ und $\pm 0^{\mathrm{m}} .09$; sie sind in Ansehung der geringen w. Fehler als reell zu betrachten. Das starke Ueberwiegen des positiven Vorzeichens wenigstens in der ersten Grössenabtheilung zeigt den Einfluss des Luftzustandes in dem zu erwartenden Sinne sehr deutlich. Bei dem stärksten Unterschiede von einer Grössenclasse zwischen Platte 516 und 677 handelt es sich um eine Differenz der Luftruhe von 1 und 3. Es müssen aber auch noch andere Factoren wirksam gewesen sein, wie das Beispiel der Platten 454 und 614 lehrt, die sich aber vorläufig noch jeder Controlle entziehen und zum Theil gewiss physiologischer Natur sein werden. In Falle der Platten $5: 6$ und 677 wird nichts anderes ubrig bleiben, als später die sternärmere Platte neu aufzunehmen und auszumessen.

Von besonderem Interesse ist nun das Verhalten der $b$. In der ersten Grössenabtheilung sind dieselben positiv zu erwarten; was aber nicht deutlich ausgesprochen ist, zudem sind sie nur wenig grösser als ihre w. Fehler, die im Mittel \pm 0.0 I betragen. Es folgt hieraus die wichtige Thatsache, dass es Dr. Schwassmann wirklich gelungen ist, durch seine Schätzungsmethode den Einfluss des Abstandes von der Plattenmitte bei den schwächeren Sternen fast gänzlich zu eliminiren, wie dies beabsichtigt worden war.

Bei den helleren Sternen ist dies nicht gelungen; bei ihnen tritt das $z u$ erwartende negative Vorzeichen der $b$ allein auf. Im Mittel betragen die $b-0^{m}$. 7 und $-0^{\mathrm{m}}$ ro mit den w. Fehlern der einzelnen $b \pm 0^{\mathrm{m}} \mathrm{o}_{2}$ resp. $\pm 0^{\mathrm{m}} \circ 0_{3}$, d. h. die Grössen sind in den Ecken der Platten um etwa 0.6 bis 0.8 Grössenclassen heller geschätzt als in der Mitte. Während also bei den Sternen schwächer als 9.2 das Grössenmaterial als homogen auf einer Platte zu betrachten ist, ist dies bei den helleren Sternen nicht der Fall.

Bei Gelegenheit dieser Vergleichungen habe ich auch den w. Fehler der einzelnen Grössenschätzungen bestimmt. Derselbe beträgt für die schwächeren Sterne $\pm 0^{\mathrm{m}}{ }_{\mathrm{r}}$, für die helleren $\pm 0^{m_{2}}$. Aus der Vergleichung der Grössen- 
schätzungen der BD. mit den Potsdamer photometrischen Zonen (Publ. des Astrophys. Obs. Band 9) ist als w. Fehler der ersteren $\pm 0 \frac{m}{2}$ ermittelt worden; demnach sind die Grössenschätzungen der Himmelskarte bei den allein in Frage tretenden schwächeren Sternen von der gleichen Genauigkeit wie die in der BD. gegebenen Mittelzahlen.

Für Miss Everett stellt sich die entsprechende Untersuchung folgendermaassen:

\begin{tabular}{|c|c|c|c|c|c|c|c|c|c|}
\hline \multirow[b]{2}{*}{ Platten Nir. } & \multicolumn{3}{|c|}{ Grösse I I bis 9.3} & \multicolumn{3}{|c|}{ Grösse 9.2 bis 8.5} & \multicolumn{3}{|c|}{ Grösse 8.4 bis 7.5} \\
\hline & $a$ & $\dot{b}$ & $n$ & $a$ & $b$ & $n$ & $a$ & $b$ & $n$ \\
\hline $05-1062$ & 0.14 & $+0.1 \mathrm{I}$ & 103 & 0.13 & -0.3 & 26 & - & - & 4 \\
\hline-1062 & +0.15 & +0.06 & 87 & +0.08 & -0.05 & 19 & - & - & $\mathbf{I}$ \\
\hline$-106_{3}$ & +0.15 & +0.08 & 107 & +0.06 & -0.02 & 17 & - & - & 6 \\
\hline 1063 & +0.29 & +0.09 & 86 & +0.12 & -0.03 & 25 & -0.06 & -0.14 & 8 \\
\hline .064 & $-0.5^{\circ}$ & +0.07 & 89 & -0.29 & -0.07 & 14 & -0.33 & $-0.1 \mathrm{I}$ & 10 \\
\hline-1064 & -0.55 & +0.05 & 133 & -0.15 & -0.08 & 19 & - & - & 4 \\
\hline 065 & $-0.3^{6}$ & +0.09 & I 25 & -0.39 & -0.05 & 26 & -0.42 & -0.05 & 15 \\
\hline-1065 & +0.03 & +0.08 & 97 & +0.09 & -0.06 & 19 & - & - & $=$ \\
\hline-1066 & +0.00 & +0.06 & 105 & -0.02 & -0.12 & 17 & - & - & 6 \\
\hline-1066 & +0.05 & +0.06 & I 34 & +0.17 & -0.07 & 23 & +0.04 & -0.18 & 8 \\
\hline $062-918$ & -0.26 & +0.10 & 82 & -0.24 & -0.06 & 9 & - & -- & 2 \\
\hline $106_{3}-918$ & -0.27 & +0.08 & I I 4 & -0.35 & -0.06 & 16 & - & - & 5 \\
\hline $106_{3}-919$ & +0.19 & +0.07 & 144 & +0.18 & -0.03 & 16 & +0.06 & -0.09 & 13 \\
\hline $1064-919$ & $+0.3^{8}$ & +0.05 & 113 & +0.59 & -0.02 & 35 & +0.59 & 0.00 & 19 \\
\hline $1064-920$ & +0.35 & +0.06 & 120 & +0.48 & -0.07 & 35 & $+0.3 I$ & -0.15 & I 2 \\
\hline $1065-920$ & +0.26 & +0.07 & 132 & +0.32 & -0.06 & 21 & +0.20 & -0.11 & 10 \\
\hline $066-922$ & $+0.1 \mathrm{x}$ & +0.05 & 142 & +0.20 & -0.07 & 34 & +0.17 & -0.08 & 17 \\
\hline
\end{tabular}

Die constanten Abweichungen der Grössenschätzungen sind hier nur halb so gross wie die entsprechenden im I. Bande. Die $a$ betragen im Mittel für die drei Abtheilungen $0.24,0.23$ und $0^{\mathrm{m}} .24$ mit den $w$. Fehlern der einzelnen $a$ von $\pm 0.02, \pm 0{ }^{\mathrm{m}} .04$ und \pm 0.07 . Ich glaube, dass der Unterschied zwischen den $a$ im I. und II. Bande nicht ein zufälliger ist, sondern darauf beruht, dass Miss Everett eine sehr grosse zeitliche Gleichförmigkeit ihrer Grössenschätzungen erreicht hat, wie dies auch durch andere Untersuchungen bestätigt wird.

Abgesehen hiervon zeigt sich der Einfluss des Luftzustandes in den a genau so wie bei Schwassmann, indem das positive Vorzeichen stark überwiegt. Dass dieser Einfluss bei beiden Beobachtern nicht noch stärker hervortritt, wird hauptsächlich daran liegen, dass in manchen Fällen der Unterschied des Luftzustandes bei den beiden verglichenen Platten nur sehr gering ist, oder sogar, da er doch nur auf Taxirung beruht, im unrichtigen Sinne angegeben ist.

In Betreff der $b$ tritt bei den Grössenschätzungen von Everett eine wesentliche Verschiedenheit gegenüber den Schwassmann'schen auf, insofern als auch in der Abtheilung der schwachen Sterne das Vorzeichen der $b$ ausnahmslos positiv ist. Im Mittel ist $b=+0 \mathrm{~m} .07, \mathrm{~d}, \mathrm{~h}$. es ist hier nicht gelungen, die beabsichtigte Homogenität der Grössen. schätzungen auf ein und derselben Platte zu erreichen. Die schwachen Sterne sind am Rande der Platte um etwa $0^{\mathrm{m}} .6$ schwächer geschätzt als in der Mitte. Bemerkenswerth ist aber die zeitliche Constanz der Auffassung, wie die sehr gute Uebereinstimmung der $b$ untereinander lehrt. Der wahrscheinliche Fehler der $b$ beträgt im Mittel nur $\pm 0^{\mathrm{m}}$ or .

Bei den beiden anderen Grössenabtheilungen herrscht wieder vollständige Uebereinstimmung mit Schwassmann. Die $b$ werden im Mittel - $0^{\mathrm{m}} .06$ und $-0^{\mathrm{m}}$ ro, d. h. im II. Bande sind genau wie im I. Bande die helleren Sterne als $9 \mathbf{m}_{2}$ am Rande um etwa $0 .{ }^{\mathrm{m}} 6$ bis 0.98 heller geschätzt als in der Mitte.

Die zufälligen Abweichungen der Grössenschätzungen sind im II. Bande ebenfalls genau dieselben wie im I. Bande. Die w. Fehler einer Grössenschätzung betragen bei Everett für die drei Abtheilungen $\pm 0.2 \mathrm{r}, \pm 0 \div 19$ und \pm 0.20 .

Der Einfluss des Abstandes der Sterne von der Platten. mitte muss sich auch in der Sterndichtigkeit äussern, da die schwächsten Sterne der Mitte am Rande nicht mehr zur Abbildung gelangen werden. $\mathrm{Um}$ den numerischen Betrag dieses Einflusses genau zu ermitteln, müsste man auf einer grösseren Zahl von Platten die Sterne in den einzelnen Gitterquadraten abzählen, was aber mit einem sehr beträchtlichen Aufwand an Zeit und Arbeit verbunden wäre. Bei der Anordnung des Catalogs ist es dagegen sehr einfach, die Zahl der Sterne von gleicher $x$-Coordinate, aber durch alle $y$ hindurch zu entnehmen. Ich habe dies für eine grössere Zahl sternreicher Platten ausgeführt und gebe in der folgenden Zusammenstellung die Resultate dieser Abzählung in Procenten mit dem Argument der $x$-Coordinaten in Gitterintervallen.

\begin{tabular}{c|c|c|c}
\hline$x$-Coord. & Schwassmann & Everett & Mittel \\
\hline 0 & $8.8 \%$ & $8.0 \%$ & $8.4 \%$ \\
I & 8.1 & 8.5 & 8.3 \\
2 & 8.6 & 8.9 & 8.8 \\
3 & 9.2 & 8.7 & 9.0 \\
4 & 9.0 & 8.3 & 8.7 \\
5 & 8.3 & 7.1 & 7.7 \\
6 & 7.8 & 7.7 & 7.8
\end{tabular}




\begin{tabular}{c|c|c|c}
\hline$x$-Coord. & Schwassmann & Everett & Mittel \\
\hline 7 & $7.9 \%$ & $8.3 \%$ & $8.1 \%$ \\
8 & 7.5 & 7.9 & 7.7 \\
9 & 6.3 & 7.9 & 7.1 \\
10 & 6.5 & 6.3 & 6.4 \\
I I & 6.1 & 5.9 & 6.0 \\
12 & 5.9 & 6.0 & 6.0
\end{tabular}

Die Abnahme der Dichtigkeit nach dem Rande zu ist hieraus bereits deutlich zu erkennen; ihr numerischer Betrag lässt sich einigermaassen beurtheilen, wenn man, ähnlich wie oben bei den Grössendifferenzen, die Annahme macht, dass die Dichtigkeit proportional mit dem Abstande von der Mitte abnimmt. Bezeichnet man die Sterndichtigkeit der Gitterquadrate allgemein mit $D$, diejenige des mittleren Feldes der Platte mit $d$, so ist für eine beliebige Distanz $\varrho$

$$
D=d(\mathbf{1}-\epsilon \varrho)
$$

Die $\varrho$ lassen sich für jedes Gitterquadrat als $\sqrt{x^{2}+y^{2}}$ sehr einfach hinschreiben, und die Summation der $d$ und $d \varrho$ für jedes $x$ über die ganze Platte hinüber liefert alsdann mit Hülfe der vorstehenden Werthe I 3 Gleichungen zur Bestimmung von $d$ und $c$. Nach der Methode der kleinsten Quadrate erhält man:

$$
d=0.440 \% \pm 0.0110 \text { und } c=0.0312 \pm 0.0020 \text {. }
$$

Hieraus ergiebt sich $D$ für die Mitte des Randes zu $0.27 \%$, für die Ecken der Platten sogar zu nur $0.21 \%$, d. h. man wird mit genügender Annäherung annehmen können, dass die Sterndichtigkeit in den Plattenecken nur noch die Hälfte von derjenigen in der Mitte beträgt.

Es ist dies ein nicht gerade sehr befriedigendes $\mathrm{Re}$. sultat, und wenn dasselbe bei Festsetzung des für die Himmelskarte angenommenen Modus der Aufnahmen bekannt gewesen wäre, würde man sich vielleicht entschlossen haben, das benutzbare Gesichtsfeld etwas kleiner zu wählen und entsprechend die Zahl der Aufnahmen zu vermehren.

Ein grösserer Theil der vorstehenden Rechnungen ist von Herrn Dr. Ludendorff ausgeführt worden.

Potsdam, Astrophysikalisches Observatorium, 190I Januar.

\title{
F. Scheiner.
}

\section{Ephemeride des Planeten (327) Columbia.}

\author{
$12^{\text {h }}$ M. Z. Berlin.
}

Die in Veröff. R. I. Nr. I 3 mitgetheilte Ephemeride ist fehlerhaft und durch folgende zu ersetzen.

\begin{tabular}{|c|c|c|c|c|c|c|c|}
\hline \multicolumn{2}{|c|}{ I 901} & \multicolumn{2}{|r|}{$\alpha$} & \multicolumn{2}{|r|}{$\delta$} & \multirow{2}{*}{$\frac{\log r}{0.4600}$} & \multirow{2}{*}{$\frac{\log \Delta}{0.2805}$} \\
\hline März & I 8 & $I_{1}{ }^{h}$ & $I^{m} 43^{s}$ & $+7^{\circ}$ & $4 I ! 4$ & & \\
\hline & 20. & I I & $0 \quad 2$ & 7 & 46.8 & 4598 & 2818 \\
\hline & 22 & 10 & $5^{8} \quad 24$ & 7 & 51.9 & 4597 & 2834 \\
\hline & 24 & & $5^{6} \quad 49$ & 7 & 56.5 & 45 & 2852 \\
\hline & 26 & & $\begin{array}{ll}55 & 17\end{array}$ & 8 & 0.8 & 4593 & 2872 \\
\hline & 28 & & 5349 & 8 & 4.6 & 45 & 2894 \\
\hline & 30 & & 5225 & 8 & 8.0 & 45 & 2918 \\
\hline April & $\mathbf{I}$ & & 516 & 8 & 11.0 & 8 & 2945 \\
\hline & 3 & & $495^{2}$ & 8 & 13.6 & 4587 & 2973 \\
\hline & 5 & & $48 \quad 43$ & 8 & I 5.8 & 4585 & 3003 \\
\hline & 7 & 10 & $47 \quad 39$ & +8 & 17.4 & $0.45^{84}$ & 0.3035 \\
\hline
\end{tabular}

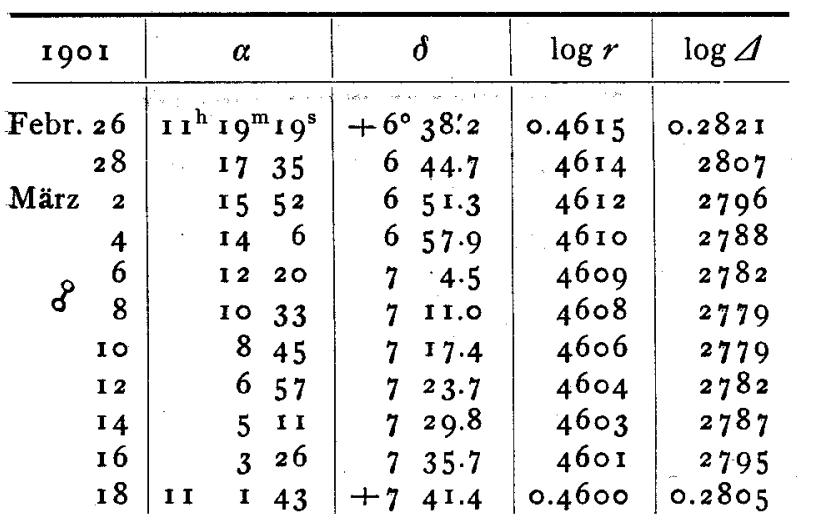

Breslau, i 90 r Febr. 20.

Grösse $=$ 13.2. RA. $\pm \mathbf{I}^{\mathrm{m}}$, Decl. $\mp 6: 0$.

P. Neugebauer.

\section{Beobachtungen der Helligkeit von (433) Eros.}

Linienschiffsähnrich Rudolf Höhl hat gestern Abend Eros wie folgt beobachtet:

I $90 \mathrm{I}$ Febr. 14. Instr. $6 \mathrm{z}$. Refr. Vergr. 50.

$$
\begin{array}{ll}
\text { Bezeichnung: } & a=\mathrm{BD} .+199^{9} 74699^{\mathrm{m}} 3 \\
& b=(433) \text { Eros } \\
& c=\mathrm{BD} .+19^{9} 74^{8} 99^{\mathrm{m}} \cdot 5 \\
& d=\mathrm{BD} .+199^{\circ} 7499 \cdot 5
\end{array}
$$

$6^{\mathrm{h}} 35^{\mathrm{m}} a b$ und $b$ i $c$ und $c d$

$7 \circ a b$ und $b$ i $c$ und $c d$ $7^{\mathrm{h}}{ }^{\mathrm{m}} 3^{\mathrm{m}}$ bis $7^{\mathrm{h}} 24^{\mathrm{m}}$ am Ringmikrometer Planet (433) Eros mit $*$ BD. + $1997426^{\mathrm{m}} \cdot 5$ beobachtet, ohne eine Helligkeitsänderung wahrzunehmen; dann

$7^{\mathrm{h}} 3^{2^{\mathrm{m}}}$ auf den ersten Blick die Lichtabnahme von Eros erkannt und beobachtet:

$$
\begin{aligned}
& 7^{\mathrm{h}} 3^{2^{\mathrm{m}}} a_{3} b \text { und } c_{\mathbf{1}} b \\
& 75^{8} \quad a_{4} b \text { und } c_{2} b \\
& 8 \quad 25 \quad a 3 b \text { und } c 2 b
\end{aligned}
$$

Ich finde hieraus I Stufe $=0^{\mathrm{m}} \mathrm{I}_{4}$ und weiter: 